\title{
Effect of Ventricular Shock Strength on Cardiac Hemodynamics
}

\author{
TAKASHI TOKANO, M.D., DAVID BACH, M.D., JASON CHANG, \\ JAMES DAVIS, M.D., JOSEPH J. SOUZA, M.D., ADAM ZIVIN, M.D., \\ BRADLEY P. KNIGHT, M.D., RAJIVA GOYAL, M.D., K. CHING MAN, D.O., \\ FRED MORADY, M.D., and S. ADAM STRICKBERGER, M.D. \\ From the Division of Cardiology, Department of Internal Medicine, \\ University of Michigan Medical Center, Ann Arbor, Michigan
}

\begin{abstract}
Ventricular Defibrillation and Cardiac Function. Introduction: The effect of implantable defibrillator shocks on cardiac hemodynamics is poorly understood. The purpose of this study was to test the hypothesis that ventricular defibrillator shocks adversely effect cardiac hemodynamics.

Methods and Results: The cardiac index was determined by calculating the mitral valve inflow with transesophogeal Doppler during nonthoracotomy defibrillator implantation in 17 patients. The cardiac index was determined before, and immediately, 1 minute, 2 minutes, and 4 minutes after shocks were delivered during defibrillation energy requirement testing with 27to 34-, 15-, 10-, 5-, 3-, or 1-J shocks. The cardiac index was also measured at the same time points after 27- to 34-, and 1-J shocks delivered during the baseline rhythm. The cardiac index decreased from $2.30 \pm 0.40 \mathrm{~L} / \mathrm{min}$ per $\mathrm{m}^{2}$ before a 27 - to $34-\mathrm{J}$ shock during defibrillation energy requirement testing to $2.14 \pm 0.45 \mathrm{~L} / \mathrm{min}$ per $\mathrm{m}^{2}$ immediately afterwards $(P=0.001)$. This effect persisted for $>\mathbf{4}$ minutes. An adverse hemodynamic effect of similar magnitude occurred after $15 \mathrm{~J}(P=0.003)$ and 10 -J shocks $(P=0.01)$, but dissipated after 4 minutes and within 2 minutes, respectively. There was a significant correlation between shock strength and the percent change in cardiac index $(r=0.3, P=0.03)$. The cardiac index decreased $14 \%$ after a 27 - to 34-J shock during the baseline rhythm $(P<0.0001)$. This effect persisted for $<4$ minutes. A 1$\mathrm{J}$ shock during the baseline rhythm did not effect the cardiac index.

Conclusion: Defibrillator shocks $>9 \mathrm{~J}$ delivered during the baseline rhythm or during defibrillation energy requirement testing result in a $10 \%$ to $15 \%$ reduction in cardiac index, whereas smaller energy shocks do not affect cardiac hemodynamics. The duration and extent of the adverse effect are proportional to the shock strength. Shock strength, and not ventricular fibrillation, appears to be most responsible for this effect. Therefore, the detrimental hemodynamic effects of high-energy shocks may be avoided when low-energy defibrillation is used. (J Cardiovasc Electrophysiol, Vol. 9, pp. 791-797, August 1998)
\end{abstract}

defibrillation threshold, implantable cardioverter defibrillator, cardiac index, cardiac output, stunning

Introduction

Ventricular implantable defibrillators frequently are programmed to deliver the maximum shock energy, irrespective of the defibrillation energy requirement. However, the effect of ventricular im-

Address for correspondence: S. Adam Strickberger, M.D., University of Michigan Medical Center, 1500 East Medical Center Dr., Ann Arbor, MI 48109-0022. Fax: 734-936-7026.

Manuscript received 23 April 1998; Accepted for publication 28 May 1998. plantable defibrillator shock strength on cardiac hemodynamics has not been well defined. ${ }^{1-5}$ Therefore, the purpose of this study was to test the hypothesis that nonthoracotomy ventricular defibrillator shocks adversely affect cardiac hemodynamics.

\section{Methods}

\section{Patient Population}

The study population consisted of 17 patients (12 men and 5 women; mean age $55 \pm 16$ years) 
undergoing implantation of a transvenous implantable cardioverter defibrillator. Eight patients had coronary artery disease, 8 patients had a nonischemic cardiomyopathy, and 1 patient had no structural heart disease. The mean left ventricular ejection fraction was $0.32 \pm 0.12$ (range 0.15 to 0.55 ), and 4 patients had an ejection fraction $>0.40$. The mean New York Heart Association Congestive Heart Failure Class was $1.9 \pm 0.7$ (range Class I to III). The baseline rhythm was sinus in 14 patients and atrial fibrillation in 3. Transesophageal Doppler echocardiography was used to determine mitral valve inflow (see following). Cardiac output equals cardiac inflow only in the absence of significant mitral regurgitation. Therefore, patients were evaluated preoperatively for mitral regurgitation with either cardiac catheterization or with two-dimensional surface echocardiography. Patients with more than minimal echocardiographically evident mitral regurgitation were not eligible for participation in the study protocol.

\section{Defibrillator System and Implantation}

All patients came to the operating room in a postabsorptive state. Therapy with antiarrhythmic medications was discontinued at least five half-lives prior to device implantation, except in eight patients in whom amiodarone therapy had been ineffective. Angiotensin-converting enzyme inhibitor therapy, calcium channel blocker therapy, beta-blocker therapy, and digoxin were continued in 11, 1, 4, and 10 patients, respectively. General anesthesia was induced, the patients were intubated, and a constant level of anesthesia was maintained throughout the implant procedure and study protocol using supplemental doses of inhalational agents, fentanyl and its derivatives, or a steroid anesthetic.

A transvenous dual coil defibrillation lead was implanted in 16 patients (models 75, 115, and 125, Cardiac Pacemakers, Inc. [CPI], St. Paul, MN, USA). A transvenous defibrillation lead with a single shocking electrode (model 6936, Medtronic Inc., Minneapolis, MN, USA) was used in one patient. The defibrillation lead was positioned in the right ventricular apex via the subclavian vein under fluoroscopic guidance.

Sixteen of the patients in this study received a defibrillator with a truncated, fixed-tilt biphasic waveform with a first phase tilt of $60 \%$ and a second phase tilt of $50 \%$ (models 1720, 1725, 1740, $1742,1743,1762$, and 1763, CPI), and one patient received a defibrillator with a first phase tilt and second phase tilt of $65 \%$ (model $7219 \mathrm{C}$, Medtronic,
Inc.). The defibrillator shell functioned as a defibrillation electrode in six patients.

\section{Study Protocol}

Written informed consent was obtained under a protocol approved by the Human Research Committee at the University of Michigan. All shocks were delivered directly from the implantable defibrillator. Shocks were delivered during the baseline rhythm and during defibrillation energy requirement testing. Shocks were delivered during the baseline rhythm to assess the isolated effect of a shock, in the absence of ventricular fibrillation, on cardiac function. In this way, the effects of a shock can be separated from the effects of ventricular fibrillation. A 1-J shock and then a 27- to 34-J shock (the maximum energy delivered by the implantable defibrillator) were delivered synchronized to the QRS complex during the baseline rhythm.

A step-down protocol was then utilized to determine the defibrillation energy requirement. The defibrillation energy requirement was defined as the lowest energy successful at converting ventricular fibrillation to the baseline rhythm. Shock energies of $15,10,5,3$, and $1.0 \mathrm{~J}$ were delivered until ventricular fibrillation failed to convert to the baseline rhythm. If the $15-\mathrm{J}$ shock was ineffective, the first shock for the subsequent induction was 20 J. A 27 - to 34-J defibrillator shock was also used to convert ventricular fibrillation to the baseline rhythm. Ventricular fibrillation was induced with ventricular pacing with a $15-\mathrm{V}$ pulse delivered every $30 \mathrm{msec}$ with a duration of $1.1 \mathrm{msec}$ for 1 to 3 seconds. The mean duration of ventricular fibrillation was $12.7 \pm 3.9$ seconds for maximum energy shocks, $9.5 \pm 0.5$ seconds for $20-\mathrm{J}$ shocks, $8.3 \pm 1.8$ seconds for $15-\mathrm{J}$ shocks, $7.4 \pm$ 1.9 seconds for $10-\mathrm{J}$ shocks, and $6.7 \pm 1.8$ seconds for shocks $<10 \mathrm{~J}(\mathrm{P}<0.0001)$. At least $5 \mathrm{~min}-$ utes was allowed to elapse between shocks delivered during the baseline rhythm and between shocks that resulted in successful defibrillation.

\section{Cardiac Output Measurement}

The cardiac output was estimated using a measure of mitral valve inflow. ${ }^{6.7}$ This technique correlates well with the cardiac output determined with conventional thermodilution techniques ${ }^{6}$ and offers the advantage of providing an assessment of beatby-beat changes in cardiac output. Conventional thermodilution techniques cannot be used to assess 
rapid changes because several measurements over approximately 2 to 3 minutes are required.

After induction of general anesthesia but before defibrillator implantation, a transesophageal echocardiography probe was inserted. The probe was positioned to optimize visualization of mitral inflow in a basal four-chamber view. ${ }^{7}$ During the protocol, the same standard view of the mitral valve was maintained to ensure consistency of the color flow Doppler interrogation. At baseline, the diameter of the mitral annulus was determined in one plane. Pulsed-wave Doppler was used to define left ventricular inflow at the level of the mitral valve annulus. ${ }^{6.7}$ Scale and sweep speed were optimized for subsequent quantitation of the velocity time integral. Based on the assumption that left ventricular inflow equals left ventricular outflow (cardiac output) in the absence of mitral regurgitation, transmitral inflow was calculated as an estimate of cardiac output using the mitral annular cross-sectional area, flow at the level of the mitral annulus, and heart rate. ${ }^{6}$ Pulsed-wave Doppler at the level of the mitral annulus was performed at baseline and was repeated immediately after each shock. All images were recorded on standard VHS videotape for subsequent off-line analysis.

Images were digitized from videotape using commercially available hardware and software (TomTec Imaging Systems, Inc., Boulder, CO, USA). The velocity time integral of left ventricular inflow was averaged for at least five consecutive cycles in all patients; 10 cycles were used for patients with atrial fibrillation. Mitral inflow velocities associated with premature ventricular complexes, post premature ventricular complexes, and ventricular paced beats were excluded from analysis. Shocks were delivered within 30 seconds of recording the baseline mitral inflow. The mean time to completion of the first mitral valve inflow determination after each shock was $19.7 \pm 10.8$ seconds.

All measurements for mitral inflow velocity time integral were determined independently by three investigators. Two of the three investigators were blinded to the shock energy and the rhythm. The mean variability of these measurements was $10.7 \%$ $\pm 6.6 \%$. The values determined by all investigators were averaged, and the means were used for analysis.

\section{Statistical Analysis}

Continuous variables are expressed as mean \pm $1 \mathrm{SD}$ and were compared using a paired or unpaired $t$-test, as appropriate. Multiple continuous variables were compared by ANOVA, and then by individual paired $t$-tests when the ANOVA result was statistically significant. The relationship between two continuous variables was assessed with a linear regression analysis. $\mathrm{P}<0.05$ was considered statistically significant.

\section{Results}

\section{Defibrillation Energy Requirements}

The mean defibrillation energy requirement was $8.6 \pm 4.8 \mathrm{~J}$ in the 17 patients. The defibrillation energy requirement was $20 \mathrm{~J}$ in 1 patient, $15 \mathrm{~J}$ in $2,10 \mathrm{~J}$ in $6,5 \mathrm{~J}$ in 6 , and $3 \mathrm{~J}$ in 2 . In one patient with a 5-J defibrillation energy requirement, a recording malfunction precluded the determination of the Doppler measurement. Because of the small number of successful defibrillations with energies $<10 \mathrm{~J}$, these data were analyzed as a single group.

\section{Effect of Shocks During Defibrillation Energy Requirement Testing}

The cardiac index decreased from $2.30 \pm 0.40$ $\mathrm{L} / \mathrm{min}$ per $\mathrm{m}^{2}$ before a 27 - to $34-\mathrm{J}$ shock $(\mathrm{n}=16)$ during defibrillation energy requirement testing to $2.14 \pm 0.45 \mathrm{~L} / \mathrm{min}$ per $\mathrm{m}^{2}$ immediately after the shock ( $\mathrm{P}=0.001$; Figs. 1 and 2$)$. The cardiac index remained depressed for $>4$ minutes $(\mathrm{P}<0.01)$. After a $15-\mathrm{J}$ shock $(\mathrm{n}=17)$ during defibrillation energy requirement testing, the cardiac index decreased from $2.32 \pm 0.44 \mathrm{~L} / \mathrm{min}$ per $\mathrm{m}^{2}$ to $2.17 \pm$ $0.41 \mathrm{~L} / \mathrm{min}$ per $\mathrm{m}^{2}$ (Table $1 ; \mathrm{P}<0.01$ ), and remained depressed for $>4$ minutes $(\mathrm{P}=0.04)$. A $10-\mathrm{J}$ shock $(\mathrm{n}=14)$ resulted in an immediate decrease of $6 \%$ in the cardiac index that reversed within 2 minutes (Table $1 ; \mathrm{P}=0.02$ ). Successful defibrillation with shocks $<10 \mathrm{~J}(\mathrm{n}=9)$ was not associated with a decreased cardiac index (Table $1 ; \mathrm{P}=0.8$ ). With shocks of $10 \mathrm{~J}$ and greater, the nadir of the cardiac index was noted 1 minute after the defibrillation shock (Table 1). The percent change in cardiac index correlated negatively with shock strength (Fig. 3; r = 0.3, P = 0.03). The change in cardiac index associated did not correlate with the duration of ventricular fibrillation or with the ejection fraction.

\section{Effect of Shocks During Baseline Rhythm}

A 27- to 34-J shock synchronized to the QRS complex during the patients' baseline rhythm ( $\mathrm{n}=$ 14) resulted in the cardiac index decreasing from 


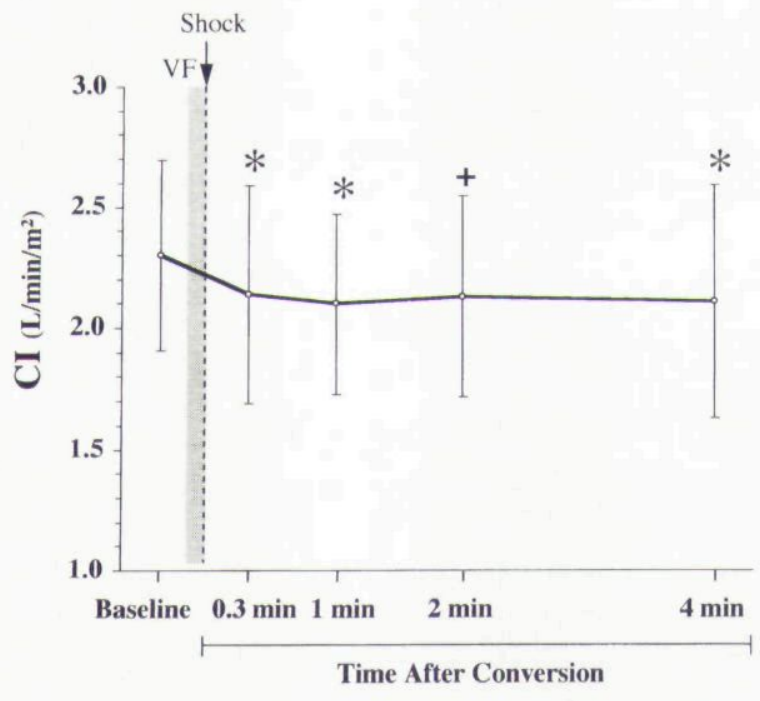

Figure 1. Cardiac index and 27-to 34-J shocks. The mean effects of a 27- to 34-J shock delivered during defibrillation energy requirement testing are shown. Cardiac index $(\mathrm{CI})$ is represented on the $y$-axis, and time is represented on the $x$-axis. $* P<0.01 ;+P<0.05$.

$2.32 \pm 0.33 \mathrm{~L} / \mathrm{min}$ per $\mathrm{m}^{2}$ to $2.00 \pm 0.33 \mathrm{~L} / \mathrm{min}$ per $\mathrm{m}^{2}$ immediately after the shock (Table $1 ; \mathrm{P}<$ $0.0001)$. This effect persisted for $<4$ minutes. A 1-J shock delivered during the baseline rhythm (n =15) did not affect the cardiac index (Table 1). The relative magnitude of change in the stroke volume was not different after a 27 - to 34-J shock delivered to treat ventricular fibrillation or when delivered during the baseline rhythm $(11.7 \% \pm$ $6.4 \%$ vs $11.6 \% \pm 5.8 \%, \mathrm{P}=1.0$ ).

The effect of defibrillation on stroke volume was nearly identical to the changes noted in car- diac index (Table 2). Heart rate was largely unaffected by the defibrillation shocks (Table 3 ).

\section{Discussion}

\section{Major Findings}

Biphasic implantable defibrillator shocks of 10 $\mathbf{J}$ or more delivered during the baseline rhythm or when used to terminate ventricular fibrillation result in a $10 \%$ to $15 \%$ reduction of cardiac index, which occurs secondary to a reduction in stroke volume. With 27- to $34-\mathrm{J}$ shocks delivered during defibrillation energy requirement testing, the cardiac index returns to normal after 4 minutes, whereas when 27- to 34-J shocks are delivered during the baseline rhythm, the adverse hemodynamic effect dissipates within 4 minutes. The duration and extent of the adverse hemodynamic effect are proportional to the shock strength.

\section{Mechanism of Shock Effect}

The detrimental hemodynamic effect of a ventricular defibrillator shock appears to be due to the shock itself, and not to ventricular fibrillation. Because a similar degree of ventricular stunning was noted after shocks delivered during the baseline rhythm as with shocks that terminated ventricular fibrillation, one must conclude that the shocks cause the stunning. This statement is supported by the fact that 27- to 34-J shocks delivered during ventricular fibrillation or during the baseline rhythm resulted in similar reduction in the cardiac index, although the adverse hemodynamic effect of shocks

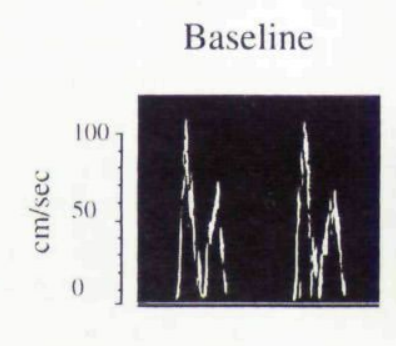

VTI $(\mathrm{cm})$
14.1
$35 \mathrm{sec}$

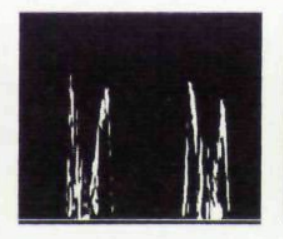

12.0
$1 \mathrm{~min}$

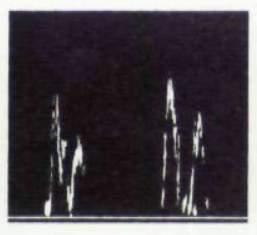

11.7
$2 \mathrm{~min}$

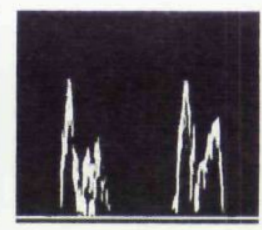

11.2
$4 \min$

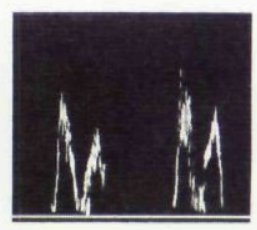

$1 \mathrm{sec}$

12.1

Figure 2. Doppler signal during defibrillation energy requirement testing. An example of the Doppler signals $(\mathrm{cm} / \mathrm{sec}) \mathrm{ob}$ tained after a 29-J shock was delivered during defibrillation energy requirement testing is shown. The baseline signal is on the left, followed by the signals obtained 35 seconds, 1 minute, 2 minutes, and 4 minutes after the shock. The mitral valve velocity time integral (VTI) value is recorded directly beneath each Doppler signal. In this example, a $14 \%$ reduction of the mitral valve velocity time integral was observed 35 seconds after the shock. The nadir of the mitral valve velocity time integral was achieved 2 minutes after the shock. 
TABLE 1

Cardiac Index Results

\begin{tabular}{|c|c|c|c|c|c|c|}
\hline & \multirow[b]{2}{*}{$\mathbf{n}$} & \multirow[b]{2}{*}{ Baseline } & \multicolumn{4}{|c|}{ Time After Shocks } \\
\hline & & & $20 \pm 11$ sec & $1 \mathrm{~min}$ & $2 \mathrm{~min}$ & $4 \mathrm{~min}$ \\
\hline \multicolumn{7}{|c|}{ DER testing: $\mathrm{CI}\left(\mathrm{L} / \mathrm{min} / \mathrm{m}^{2}\right)$} \\
\hline $27-34 \mathrm{~J}$ & 16 & $2.30 \pm 0.40$ & $2.14 \pm 0.45^{*}$ & $2.10 \pm 0.37 *$ & $2.13 \pm 0.41 \dagger$ & $2.11 \pm 0.48 *$ \\
\hline $15 \mathrm{~J}$ & 17 & $2.32 \pm 0.44$ & $2.17 \pm 0.41 *$ & $2.13 \pm 0.43^{*}$ & $2.13 \pm 0.47 *$ & $2.16 \pm 0.48 \dagger$ \\
\hline $10 \mathrm{~J}$ & 14 & $2.14 \pm 0.50$ & $2.03 \pm 0.54 \dagger$ & $1.99 \pm 0.51 \dagger$ & $2.04 \pm 0.55$ & $2.00 \pm 0.59$ \\
\hline$<10 \mathrm{~J}$ & 9 & $2.00 \pm 0.46$ & $2.04 \pm 0.46$ & $2.00 \pm 0.44$ & $1.98 \pm 0.47$ & $2.00 \pm 0.46$ \\
\hline \multicolumn{7}{|c|}{$\mathrm{SR}: \mathrm{CI}\left(\mathrm{L} / \mathrm{min} / \mathrm{m}^{2}\right)$} \\
\hline $27-34 \mathrm{~J}$ & 14 & $2.32 \pm 0.33$ & $2.00 \pm 0.33^{*}$ & $2.14 \pm 0.33^{*}$ & $2.16 \pm 0.34 *$ & $2.31 \pm 0.16$ \\
\hline $1 \mathrm{~J}$ & 15 & $2.22 \pm 0.40$ & $2.27 \pm 0.45$ & $2.18 \pm 0.36$ & $2.33 \pm 0.52$ & $2.23 \pm 0.43$ \\
\hline
\end{tabular}

$\mathrm{CI}=$ cardiac index $; \mathrm{DER}=$ defibrillation energy requirement; $\mathrm{SR}=$ baseline rhythm.

$* \mathrm{P}<0.01 ; \dagger \mathrm{P}<0.05$.

delivered during defibrillation testing lasted longer than the hemodynamic effect of shocks delivered during the baseline rhythm. If the stunning effect was primarily due to ventricular fibrillation, changes in cardiac index with shocks delivered during the baseline rhythm would not be expected.

The influence of ventricular fibrillation on these results may be difficult to separate from the effect of shocks because larger shocks required longer charge times and, hence, longer durations of ventricular fibrillation. At a minimum, one can conclude that the shorter duration of ventricular fibrillation associated with shock energies $<10 \mathrm{~J}$ are not long enough to affect cardiac hemodynamics. This supports the concept that shock strength and, to a lesser extent, ventricular fibrillation duration are responsible for this observation.

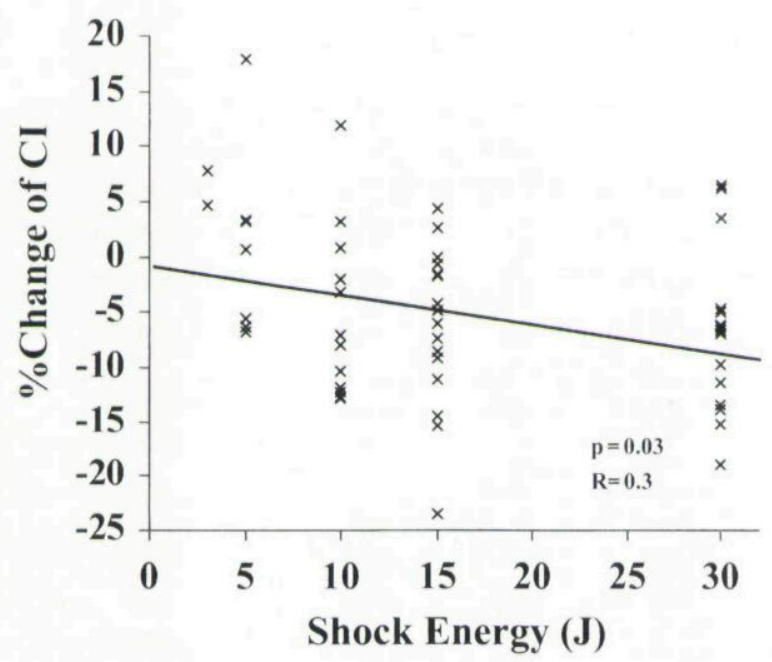

Figure 3. Shock strength and cardiac index. A significant relationship between shock strength and the percent change in the cardiac index $(C I)$ was noted. Individual patient data are denoted by an $X$.

\section{Doppler Cardiac Output Technique}

The cardiac output in the present study was estimated using a Doppler measure of mitral valve inflow. ${ }^{6,7}$ The measurements were made independently by three investigators, and the mean values were associated with an interobserver variability of $10 \%$. The mean paired data were compared and significant changes were noted. Additionally, when the data from each individual observer were analyzed independently, similar findings were obtained.

A possible limitation of the Doppler technique is that the mitral annular orifice is known to be "saddle-shaped" and to change throughout the cardiac cycle. Although using a simplified assumption of a circular annular shape may result in errors in the absolute quantitation of cardiac output, any such errors should be constant among individual patients and differences between shocks should still reflect true differences in cardiac output.

\section{Previous Studies}

The results of animal studies suggested that external and epicardial shocks synchronized to the QRS complex are associated with reduced systolic and diastolic function. ${ }^{8-11}$ More recently, the severity of systolic and diastolic dysfunction noted after defibrillation was found to be related to the shock strength in an animal model. ${ }^{12}$ In humans, transthoracic shocks given to treat ventricular tachycardia or atrial fibrillation are associated with systolic and diastolic dysfunction. ${ }^{13}$ When ventricular tachycardia was terminated with burst pacing, a change in cardiac performance was not observed. ${ }^{13}$ These results support the concept that shocks, regardless of the heart rhythm, adversely alter ventricular function.

The effect of implantable defibrillator shocks on cardiac hemodynamics in humans has varied. ${ }^{1-5}$ The inconsistent findings of the previous 
TABLE 2

Stroke Volume Results

\begin{tabular}{|c|c|c|c|c|c|c|}
\hline & \multirow[b]{2}{*}{$\mathbf{n}$} & \multirow[b]{2}{*}{ Baseline } & \multicolumn{4}{|c|}{ Time After Shocks } \\
\hline & & & $20 \pm 11 \mathrm{sec}$ & $1 \mathrm{~min}$ & $2 \mathrm{~min}$ & $4 \mathrm{~min}$ \\
\hline \multicolumn{7}{|c|}{ DER testing: SV (mL) } \\
\hline $27-34 \mathrm{~J}$ & 16 & $67.9 \pm 17.1$ & $60.2 \pm 17.0^{*}$ & $61.3 \pm 15.0^{*}$ & $62.1 \pm 14.8^{*}$ & $57.9 \pm 15.6^{*}$ \\
\hline $15 \mathrm{~J}$ & 17 & $71.1 \pm 17.8$ & $62.7 \pm 17.8^{*}$ & $64.2 \pm 18.3^{*}$ & $64.5 \pm 16.8 *$ & $62.4 \pm 15.1$ \\
\hline $10 \mathrm{~J}$ & 14 & $64.7 \pm 15.3$ & $56.6 \pm 14.2 *$ & $60.0 \pm 13.9 \dagger$ & $61.9 \pm 12.7$ & $59.2 \pm 13.1$ \\
\hline$<10 \mathrm{~J}$ & 9 & $57.3 \pm 12.0$ & $57.8 \pm 12.3$ & $55.7 \pm 13.5$ & $57.5 \pm 13.7$ & $58.0 \pm 16.5$ \\
\hline \multicolumn{7}{|l|}{ SR: SV (mL) } \\
\hline $27-34 \mathrm{~J}$ & 14 & $68.0 \pm 14.4$ & $60.2 \pm 14.1^{*}$ & $64.3 \pm 12.3 \dagger$ & $64.1 \pm 15.0$ & $66.3 \pm 13.7$ \\
\hline $1 \mathrm{~J}$ & 15 & $66.2 \pm 13.5$ & $64.4 \pm 16.9$ & $62.2 \pm 16.4$ & $64.2 \pm 16.3$ & $59.7 \pm 18.2$ \\
\hline
\end{tabular}

$\mathrm{DER}=$ defibrillation energy requirement; $\mathrm{SR}=$ baseline rhythm; $\mathrm{SV}=$ stroke volume .

$* \mathrm{P}<0.01 ; \div \mathrm{P}<0.05$.

reports probably are due to several reasons, including the use of different waveforms and lead systems, small sample sizes, noninstantaneous techniques for measuring stroke volume, and inconsistent reporting of shock strengths..$^{1-5}$ Two previous studies have assessed the effect of implantable defibrillator shocks on cardiac hemodynamics using a transvenous lead system. ${ }^{4,5}$ The results of these two studies differ significantly from those of the present study. One of the studies combined data from patients with monophasic and biphasic waveforms and epicardial and transvenous lead systems, did not include shock strength data, assessed stroke volume with a noninstantaneous thermodilution technique, and did not observe an effect of shocks on cardiac hemodynamics. ${ }^{5}$ The second study largely used the same biphasic waveform and transvenous lead system in all patients. ${ }^{4}$ This study observed that shocks decrease cardiac hemodynamics, but the study design incorporated only a single measurement in each patient at a nonuniform time between 2 and 5 minutes after each shock. ${ }^{4}$ Furthermore, shock strengths were not reported. ${ }^{4}$

\section{Limitations}

A limitation of this study is that the absolute duration of ventricular fibrillation varied depending upon the shock strength. It may be that a longer duration of ventricular fibrillation results in a more profound effect on cardiac index. If so, it is unlikely that a similar negative inotropic effect would have occurred after a 27 - to $34-\mathrm{J}$ shock delivered during the baseline rhythm as during defibrillation energy requirement testing. A second limitation is that the mitral valve Doppler signals were only recorded up to 4 minutes after each shock. Therefore, the total duration of the adverse hemodynamic effect of high-energy shocks during ventricular fibrillation was not determined. Finally, for shocks delivered during the baseline rhythm, the minimum shock energy associated with ventricular stunning cannot be determined from this study.

\section{Clinical Implications}

The results of this study have several implications for defibrillator implantation, defibrillator programming, and defibrillator development. First,

TABLE 3

Heart Rate Results

\begin{tabular}{|c|c|c|c|c|c|c|}
\hline & \multirow[b]{2}{*}{$\mathbf{n}$} & \multirow[b]{2}{*}{ Baseline } & \multicolumn{4}{|c|}{ Time After Shocks } \\
\hline & & & $20 \pm 11 \mathrm{sec}$ & $1 \mathrm{~min}$ & $2 \mathrm{~min}$ & $4 \mathrm{~min}$ \\
\hline \multicolumn{7}{|c|}{ DER testing: HR (beats/min) } \\
\hline $27-34 \mathrm{~J}$ & 16 & $70.4 \pm 15.2$ & $73.9 \pm 17.5$ & $70.9 \pm 15.3$ & $72.5 \pm 14.0$ & $75.0 \pm 15.1$ \\
\hline $15 \mathrm{~J}$ & 17 & $67.3 \pm 13.3$ & $72.2 \pm 17.9 \dagger$ & $68.9 \pm 14.5$ & $69.4 \pm 13.3$ & $71.7 \pm 12.4$ \\
\hline $10 \mathrm{~J}$ & 14 & $68.2 \pm 11.7$ & $73.5 \pm 13.8^{*}$ & $67.8 \pm 10.5$ & $67.5 \pm 10.1$ & $68.7 \pm 10.4$ \\
\hline$<10 \mathrm{~J}$ & 9 & $67.2 \pm 9.7$ & $67.9 \pm 10.9$ & $69.4 \pm 10.3$ & $68.8 \pm 9.1$ & $68.8 \pm 11.2$ \\
\hline \multicolumn{7}{|c|}{ SR: HR (beats/min) } \\
\hline $27-34 \mathrm{~J}$ & 14 & $71.2 \pm 15.6$ & $69.1 \pm 13.4$ & $69.0 \pm 15.0$ & $72.7 \pm 13.0$ & $68.1 \pm 12.0$ \\
\hline $1 \mathrm{~J}$ & 15 & $68.2 \pm 15.6$ & $71.6 \pm 13.1$ & $72.0 \pm 14.3$ & $75.2 \pm 15.7$ & $81.0 \pm 19.1$ \\
\hline
\end{tabular}

$\mathrm{DER}=$ defibrillation energy requirement; $\mathrm{HR}=$ heart rate; $\mathrm{SR}=$ baseline rhythm.

$* \mathrm{P}<0.01 ; \dagger \mathrm{P}<0.05$. 
these results suggest that defibrillators should not be routinely programmed to the maximum available energy. Lower-energy shocks that consistently result in successful defibrillation are likely to be associated with improved postshock hemodynamics. In the present study, this was demonstrated to be true with shocks delivered during the baseline rhythm and during ventricular fibrillation. The same is probably also true when defibrillator shocks are used to treat ventricular tachycardia and supraventricular tachycardias. Given that ventricular and supraventricular tachycardias are sometimes treated in the ventricular fibrillation zone of the implantable defibrillator, patients probably also receive highoutput shocks for these arrhythmias. Therefore, irrespective of the rhythm being treated by the defibrillator, if an adequate defibrillation safety margin can be achieved with a lower-energy shock, then lower-energy defibrillation may be preferred. Second, although the mechanism of presumed defibrillation-induced congestive heart failure is not known, these data suggest the use of lower-energy shocks may prevent this phenomenon..$^{14,15}$ One may argue that a $10 \%$ reduction in cardiac index may not be important for normal patients. However, patients with implanted defibrillators typically have a low ejection fraction, an advanced New York Heart Association heart failure classification, and a reduced cardiac reserve. These frequent comorbidities suggest that the $10 \%$ reduction in cardiac index after defibrillation may be clinically important, and that the current industry direction toward lower maximum energy defibrillators is appropriate. Additionally, lower-energy defibrillation results in a shorter arrhythmia duration, which may limit the ischemic effects of ventricular fibrillation. Finally, these data have implications for the waiting time between inductions of ventricular fibrillation used to determine the defibrillation energy requirement. During defibrillation energy requirement testing using a step-down protocol, a minimum of 4 minutes should be allowed to elapse between inductions of ventricular fibrillation.

\section{References}

1. Antunes ML, Spotnitz HM, Livelli FD, et al: Effect of electrophysiological testing on ejection fraction during cardioverter/defibrillator implantation. Ann Thorac Surg 1988;45:315-318.
2. Hachenberg T, Hammel D, Möllhoff T, et al: Cardiopulmonary effects on internal cardioverter/defibrillator implantation. Acta Anaesthesiol Scand 1991; 35:626-630.

3. Stoddard MF, Redd RR, Buckingham TA, et al: Effects of electrophysiologic testing of the automatic implantable cardioverter-defibrillator on left ventricular systolic function and diastolic filling. Am Heart J 1991;122:714-719.

4. Steinbeck G, Dorwarth U, Mattke S, et al: Hemodynamic deterioration during ICD implant: Predictors of high-risk patients. Am Heart J 1994;127:1064-1067.

5. Meyer J, Möllhoff T, Seifert T, et al: Cardiac output is not affected during intraoperative testing of the automatic implantable cardioverter defibrillator. J Cardiovasc Electrophysiol 1996;7:211-216.

6. Lewis JF, Kuo LC, Nelson, JG, et al: Pulsed Doppler echocardiographic determination of stroke volume and cardiac output: Clinical validation of two new methods using the apical window. Circulation 1984;70:425-431.

7. Dennig K, Rudolph W: Mitral valve apparatus. In Roelandt JRTC, Pandian NG, eds: Multiplane Transesophageal Echocardiography. Churchill Livingstone, New York, 1996, pp. 69-85.

8. Osswald S, Trouton TG, O'Nunain SS, et al: Relation between shock-related myocardial injury and defibrillation efficacy of monophasic and biphasic shocks in a canine model. Circulation 1994;90:2501-2509.

9. Laniado S, Buckley NM, Frank CW, et al: Effects of DC electric countershock on ventricular function, cation balance and endogenous norepinephrine in the dog heart. Cardiology 1974;59:253-267.

10. Kerber RE, Martins JB, Gascho JA, et al: Effect of direct-current countershocks on regional myocardial contractility and perfusion. Experimental studies. Circulation 1981;63:323-332.

11. Ditchey RV, LeWinter MM: Effects of direct-current electrical shocks on systolic and diastolic left ventricular function in dogs. Am Heart J 1983;105:727-731.

12. Xie J, Weil MH, Sun S, et al: High-energy defibrillation increases the severity of postresuscitation myocardial dysfunction. Circulation 1997;96:683-688.

13. Stoddard MF, Labovitz AJ, Stevens LL, et al: Effects of electrophysiologic studies resulting in electrical countershock or burst pacing on left ventricular systolic and diastolic function. Am Heart J 1988;116: 364-370.

14. Frame R, Brodman R, Gross J, et al: Initial experience with transvenous implantable cardioverter defibrillator lead systems: Operative morbidity and mortality. PACE 1993;16:149-152.

15. Mansourati J, Larlet JM, Salaun G, et al: Safety of high energy internal cardioversion for atrial fibrillation. PACE 1997;20:1919-1923. 
This document is a scanned copy of a printed document. No warranty is given about the accuracy of the copy. Users should refer to the original published version of the material. 